\title{
Effect of Self Preferred Romantic Music after Physical Exertion in Males
}

\author{
Sanjeev Nara \\ Department of Biomedical \\ Engineering \\ Deenbandhu Chhotu Ram \\ University of Science and \\ Technology, Murthal
}

\author{
Manvinder Kaur \\ Department of Biomedical \\ Engineering \\ Deenbandhu Chhotu Ram \\ University of Science and \\ Technology, Murthal
}

\author{
Saurav Dutta \\ Department of Biomedical \\ Engineering \\ Prabhu Dayal Memorial College \\ of Engineering, Bahadurgarh
}

\begin{abstract}
Romantic music is considered to promote relaxation and reduce stress. Past researchers reveals that different types of music varying in tempo can have physiological effects on the body. To examine the effects of Romantic music after physical exertion, 15 male students performed a stressful, cardiovascular exercise. After performing the running exercise, participants were made listened to their preferred romantic bollywood music. The physiological effects of romantic music promoting relaxation are indicated by changes in Heart rate variability (HRV). Specifically, the relative change in low to high frequency (LF/HF) ratio component of heart rate variability proven to be a non invasive technique was used to indicate physiological changes occurring in the Autonomic nervous system (ANS). The results obtained demonstrate the effects of romantic music after physical exertion based on individual preference for the song.
\end{abstract}

\section{Keywords}

Romantic music, Heart rate variability, Autonomic Nervous system, $\mathrm{LF} / \mathrm{HF}$ ratio

\section{INTRODUCTION \& BACKGROUND}

Listening to music has long been known to affect the human body involving psychological, emotional, neurological and cardiovascular changes. Many studies have indicated that music affects the Autonomic Nervous System (ANS), primarily through the study of Heart Rate (HR) and Heart rate Variability (HRV), as well as other measures which indicate ANS activity such as galvanic skin response (GSR) and Blood Pressure (BP) [13]. Additionally it is proposed that music may affect healing, through vibration and sound. These studies provide evidence that leads some researchers to recommend music as a form of therapy. In 1998, Chlan measured the effect of music on HR and found that a music therapy was effective in promoting cardiac relaxation [18]. In 2001, Hamel's used music in order to try and reduce HR before catheterisation. It was found that subjects who listened to music with $70-80$ beats per minute (bpm) for 20 minutes before catheterisation displayed altered autonomic, immune, endocrine and neuropeptide states compared to the control group that received treatment as usual. The altered systems resulted in decreased HR, lower metabolism and reduced RR Interval. These findings indicated that music caused a physiological relaxation state [21]. In 2002, Smolen and his team-mates used similar measures with colonoscopy as the intervention, with similar findings. Their study observed the effects of music therapy on physiological signs of anxiety among colonoscopy patients. HR and BP were measured during the procedure, both of which were reported to be significantly decreased in the experimental group which listened to self-selected music [3]. Both these studies indicated that physiological measures, predominately HR, which relate to ANS activity, were reduced in subjects who listened to music compared to controls. Many studies showed that music can be used to induce moods in experimental settings, and by extension can be used for therapeutic purposes $[1,4,6,12,19]$. It was also claimed that music can be used to improve concentration, reduce anxiety and promote relaxation [18], as well as enhance well-being and reduces stress [16]. In 2002, Panksepp and Bernatzky looked at the role of the brain in interpreting music on an emotional level, and autonomic responses to music [10]. It was also noted that gender has an influence on physiological measures, as men and women differ in their music preferences and emotional reactions to music and if the subject choose their own music then there is a stronger effect from the music rather than if it is selected by the researcher or therapist [20].

The main aim of present study is to examine the effects of self preferred romantic music after physical exertion in males. The physiological changes occurring in ANS before and after listening to music during post recovery after performing cardiovascular running exercise for about twenty minutes are indicated by changes occurring in HRV. The ANS is further divided into the sympathetic nervous system (SNS) and parasympathetic nervous system (PNS). SNS is responsible for increasing heart rate, and PNS is responsible for decreasing heart rate [2]. Both time domain and frequency domain measures of HRV can be used to indicate the changes in ANS. In present study, frequency domain analysis of HRV is performed to indicate the required changes. An increase in $\mathrm{LF} / \mathrm{HF}$ is believed to indicate sympathetic nervous system activity while a decrease indicates parasympathetic activity [5]. Therefore $\mathrm{LF} / \mathrm{HF}$ would come more close to normal value after listening to self preferred romantic music as compared to the condition when no music is there that shows faster physiological relaxation and stress reduction while listening to romantic music.

\section{METHOD}

\subsection{Participants}

Fifteen male student volunteers of age between 20-24 years were recruited as participants. In addition, all volunteers reported no hearing problems and they liked listening to Romantic Bollywood music. 


\subsection{Procedure}

Before performing the exercise, ECG measurements were obtained from each participant to indicate normal readings. After that each participant was asked to do a cardiovascular running exercise for about twenty minutes in the ground. After exercise, ECG reading was taken from them using BIOPAC MP36 system to examine post recovery when no music is there. After this set of measurements, participants again performed twenty minutes of running exercise, ECG readings were then again acquired from them while they listened to their self preferred romantic bollywood song through earphones at a comfortable listening level to indicate the changes that are occurring in ANS.

\subsection{Processing of Signals}

The Sampling rate for ECG data acquisition was 1024 samples/sec. The Processing of signals comprises of two steps- pre-processing and feature extraction. In the preprocessing step, removal of baseline drift and noise were done. A few pre-processing steps were employed to remove artifacts and noise. Firstly, 6th level wavelet decomposition was done on the signal using daubechies-db5 mother wavelet so as to remove baseline wandering. The difference between the original and the 6th level approximation signal represents the signal with eliminated baseline wandering. In the feature extraction step, detection of $\mathrm{R}$ peak and QRS complexes was done. After detecting $\mathrm{R}$ peaks, Heart Rate Variability was calculated. For Feature Extraction Pan Tompkins algorithm was used. The Pan Tompkins Algorithm is shown in Figure-1.

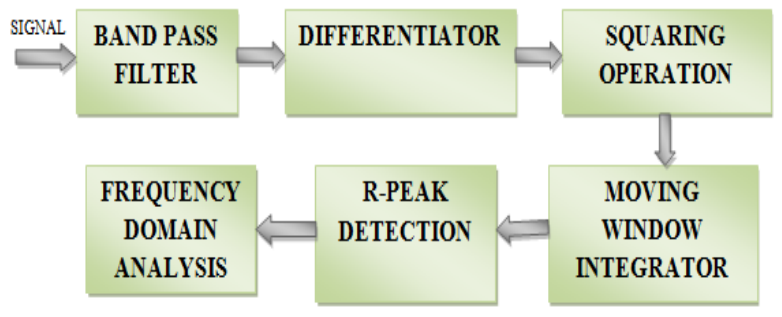

Fig-1: Pan Tompkins Algorithm

Using Pan Tompkins algorithm, the ECG signal was first passed through a low-pass and a high-pass filter in order to remove noise from the signal. Then the filtered signal was passed through derivative, squaring and window integration phases [9]. Finally, a thresholding technique was applied and the R-peaks were detected. Frequency domain measures were then calculated to quantify heart rate variability in the participants. The implementation of this algorithm on signals acquired from participants in MATLAB is shown in Figure-2.

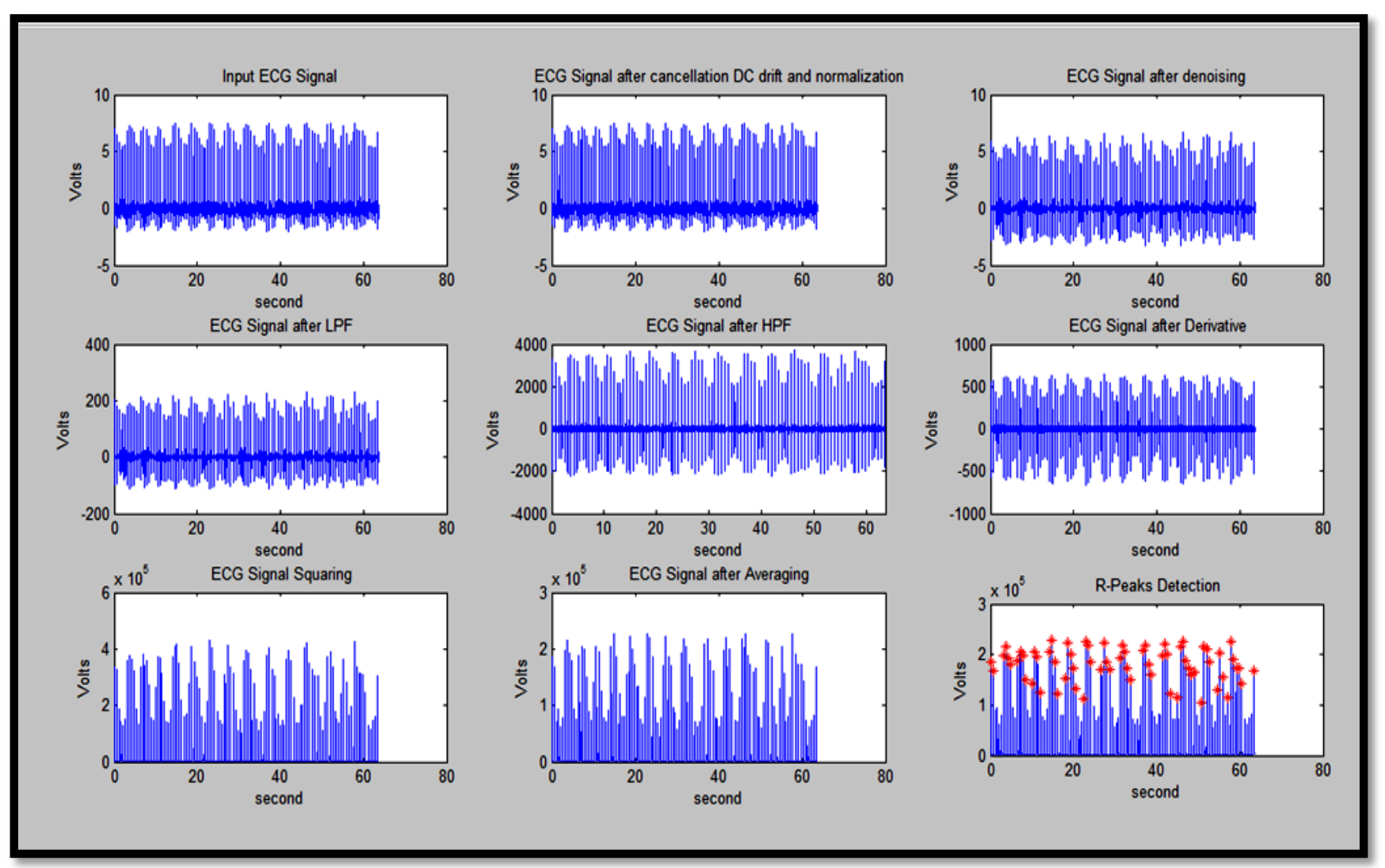

Fig-2: Implementation of PAN Tompkins Algorithm on signals acquired from Participants in MATLAB 


\section{RESULT}

The parameters calculated are LF power, HF power and ratio of LF power/ HF power. The LF power spectrum is evaluated in the range from 0.04 to $0.15 \mathrm{~Hz}$. This band can reflect both sympathetic and parasympathetic tone. It is calculated by the use of Power Spectral Density (PSD) of signal. The HF power spectrum is evaluated in the range from 0.15 to $0.4 \mathrm{~Hz}$. This band reflects parasympathetic tone. LF/HF Ratio is used to indicate balance between sympathetic and parasympathetic tone. Table- 1 shows a generalized value of LF, HF and their ratio evaluated through the spectral components of different participants

Table-1: HRV Spectral Components

\begin{tabular}{|c|c|c|c|}
\hline Category & $\begin{array}{c}\text { Low } \\
\text { Frequency } \\
\text { (Mean } \pm \text { SD) }\end{array}$ & $\begin{array}{c}\text { High } \\
\text { Frequency } \\
\text { (Mean } \pm \text { SD) }\end{array}$ & $\begin{array}{c}\text { LF/HF } \\
\text { Ratio } \\
\text { (Mean } \pm \text { SD) }\end{array}$ \\
\hline Normal & $75.19 \pm 4.08$ & $64.97 \pm 4.28$ & $1.15 \pm 0.0108$ \\
\hline No Music & $72.49 \pm 3.44$ & $62.26 \pm 3.46$ & $1.171 \pm 0.015$ \\
\hline $\begin{array}{c}\text { Romantic } \\
\text { Bollywood } \\
\text { Music }\end{array}$ & $72.74 \pm 3.73$ & $62.61 \pm 3.77$ & $1.161 \pm 0.037$ \\
\hline
\end{tabular}

From the above table it can be evaluated in post recovery period during listening to Romantic bollywood music, the LF/HF ratio comes close to the normal value that can directly be linked to parasympathetic activity indicating early relaxation than the LF/HF ratio when no music was there. For all the participants, we have consistently measured LF, HF, LF and HF ratio in normalized units which is consistent with our hypothesis. Figure- 3 below shows the result of our measurement of $\mathrm{LF} / \mathrm{HF}$ ratio of 15 participants. The blue line indicates the ratio values in normal condition; red line indicates ratio values when no music was there and green line indicates ratio values when participants were listening to romantic bollywood music.

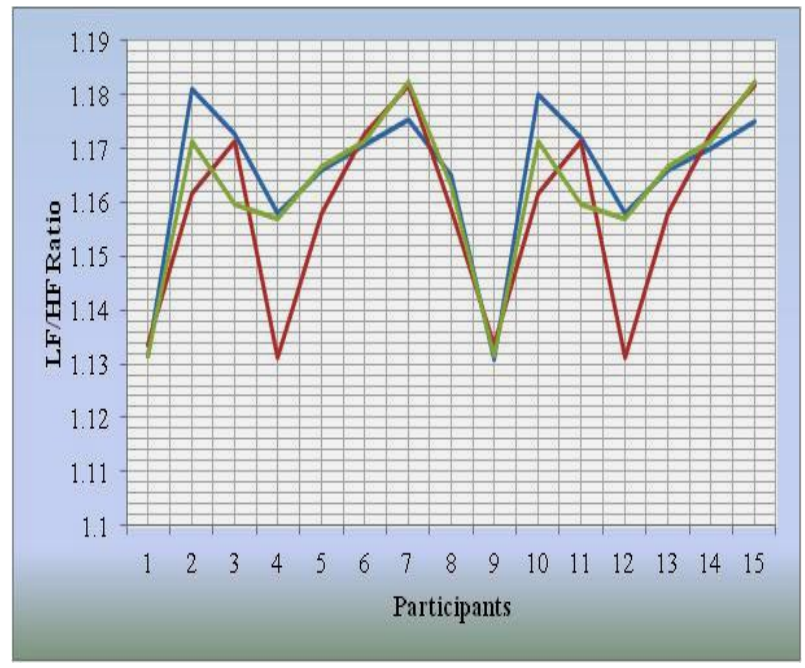

Fig-3: Measurement of LF/HF ratio of 15 participants

\section{CONCLUSION}

The Purpose of this study was to evaluate the overall effect self preferred Romantic music on Heart Rate Variability and in turn on the sympathetic and parasympathetic activity of the subject to promote relaxation after physical stress in males. Our findings indicate that listening to romantic self preferred music results in significantly reduced physical stress in males after exposure to a stressor. These findings have important role in management of anxiety or tension in male patients by music therapy. These would help the music therapist to select a particular type of romantic music for his male patient according to patient's choice. In our future study, similar experiment would be conducted on female participants to test the effect of self preferred romantic music on them in reducing stress to evaluate the significant changes that would occur due to gender difference.

\section{ACKNOWLEDGMENT}

We are extremely grateful to the Dr. Arun Garg, Director, PDM College of Pharmacy, Bahadurgarh, Haryana for allowing us to work in their lab. Gratitude would be incomplete without the mention of our student volunteers who enthusiastically supported us by giving their ECG readings under three different conditions without any hesitation.

\section{REFERENCES}

[1] A. Scott, "An Investigation into the Effects of Researcher Selected Music vs. Self Selected Music upon Muscle Tone and Mood," British School of Osteopathy, London, 2000 .

[2] B.F. Robinson, S.E. Epstein, G.D. Beiser and E. Braunwald, "Control of Heart Rate by the Autonomic Nervous System: Studies in Man on the Interrelation between Baroreceptor Mechanisms and Exercise," Circulation Research: Journal of the American Heart Association, 1966, Vol. 19, pp. 400-411

[3] D. Smolen, R. Topp, and L. Singer, "Effect of SelfSelected Music During Colonoscopy on Anxiety, Heart Rate and Blood Pressure," Applied Nursing Research, August 2002, Vol.16, pp. 126-136.

[4] G. Kreutz, S. Bongard, S. Rohrmann, V. Hodapp and D. Grebe, "Effects of Choir Singing or Listening on Secretory Immunoglobulin A, Cortisol and Emotional State," Journal of Behavioural Medicine, December 2004, pp. 623-635.

[5] G. Milicevic, "HRV Spectra and ANS in Cardiac Disease," Coll. Antropol., 2005, Vol. 29, pp. 295-300

[6] H.L. Lai and M. Good, "Music Improves Sleep Quality in Older Adults," Journal of Advanced Nursing, February 2005, pp. 234-244.

[7] J. Jiang, L. Zhou, D. Rickson and C. Jiang, "The effects of sedative and stimulative music on stress reduction depend on music preference," The Arts in Psychotherapy, Elsevier Inc., 2013, Vol. 40, pp. 201205.

[8] J. Morgan, "A Comparative Study of Music and Soft Tissue Massage on Heart Rate Variability," Master of Osteopathy Thesis, Unitec Institute of Technology, New Zealand, 2010.

[9] J. Pan and W.J. Tompkins, "A Real-time QRS Detection Algorithm," IEEE Transaction on Biomedical Engineering, March 1985, Vol. BME-32, No.3, pp. 230236.

[10] J. Panksepp and G. Bernatzky, "Emotional Sounds and the Brain: Neuro-Affective Foundations of Musical Appreciation and Behavioural Processes," 2002, Vol. 60, pp.133-155. 
[11] J. Walsh, "An Investigation into the Physiological Effects of Music of the Patients Choice Played during Osteopathic Treatment," British School of Osteopathy, London, 2000.

[12] J.A. Etzel, E.L. Johnsen, J. Dickerson, D. Tranel and R. Adolphs, "Cardiovascular and Respiratory Responses during Musical Mood Induction,” International Journal of Psychophysiology, 2005.

[13] K. Okada, A. Kurita, A., B. Takase, T. Otsuka, E. Kodani and Y. Kusama, "Effects of Music Therapy on Autonomic Nervous System Activity, Incidence of Heart Failure Events, and Plasma Cytokine and Catecholamine Levels in Elderly Patients with Cerebrovascular Disease and Dementia," International Heart Journal, 2009, Vol. 50, No. 1, pp. 95-110.

[14] K. Trochidis, D. Sears, D.L. Tran and S.M Adams, "Psychophysiological measures of emotional response to Romantic orchestral music and their musical and acoustic correlates," 9th International Symposium on Computer Music Modelling and Retrieval, Queen Mary University of London, June 2012, pp. 19-22.

[15] K. Urakawa and K. Yokoyama, "Music can Enhance Exercise-induced Sympathetic Dominancy Assessed by Heart Rate Variability," Tohoku Journal of Experimental Medicine, 2005, Vol. 206, pp. 213-218.
[16] K. J. Kemper and S.C. Danhauer, "Music as Therapy," Southern Medical Journal, 2005, pp. 282-288.

[17] L. Bernardi, C. Porta and P. Sleight, "Cardiovascular, Cerebrovascular and Respiratory Changes Induced by Different Types of Music in Musicians and NonMusicians: The Importance of Silence Heart," International Heart Journal, 2006, Vol.92, pp. 445-452.

[18] L. Chlan, "Effectiveness of a Music Therapy Intervention on Relaxation and Anxiety for Patients Receiving Ventilatory Assistance Heart \& Lung," International Heart Journal 1998, Vol. 27, No. 3, pp. 169-176.

[19] M. Malinova and M. Krusteva, "Therapeutic Effects of Music on Preterm Infants in Neonatal Intensive Care Units," Journal of Music Therapy, 2004, Vol.43, pp. 2931

[20] U.M. Nater, E. Abbruzzese, M. Krebs and U. Ehlert, "Sex Differences in Emotional and Psychophysiological Responses to Musical Stimuli," International Journal of Psychophysiology, 2006.

[21] W.J. Hamel, "Effects of Music Intervention on Anxiety in the Patient Waiting for Cardiac Catheterization," Journal of Intensive and Critical Care Nursing, 2001, Vol.17, pp. 279-285. 